Development of an innovative method to predict and to characterize the performances of Ti-6Al-4V LBW joints

Mariacira Liberini', Sara Esposito, Kambitz Reshad, Barbara Previtali, Marco Viola, and Antonino Squillace

Citation: AIP Conference Proceedings 1769, 200012 (2016); doi: 10.1063/1.4963630

View online: http://dx.doi.org/10.1063/1.4963630

View Table of Contents: http://aip.scitation.org/toc/apc/1769/1

Published by the American Institute of Physics 


\title{
Development of an Innovative Method to Predict and to Characterize the Performances of Ti-6Al-4V LBW Joints
}

\author{
Mariacira Liberini $^{1,}$ a), Sara Esposito ${ }^{1)}$, Kambitz Reshad $^{2)}$, Barbara Previtali $^{2)}$, \\ Marco Viola ${ }^{3)}$, Antonino Squillace ${ }^{1)}$ \\ ${ }^{I}$ University of Naples "Federico II", Dept. of Chemical, Materials and Industrial Production Engineering, P.le \\ Tecchio 80, 80125, Naples, Italy \\ ${ }^{2}$ Polytechnic of Milan, Mechanical Engineering Department, via La Masa 1, 20156, Milano, Italy \\ ${ }^{3}$ Sapienza University of Rome, Dept. of Computer, Control and Management Engineering, Via Ariosto 25,
} 00185 Rome, Italy.

${ }^{a)}$ mariacira.liberini@unina.it

\begin{abstract}
Every manufacturing process leaves on the surface of the piece a typical "technology signature". In particular, the laser welding leaves a feature at the edge of the weld bead called "undercut". In this work an experimental campaign has been conducted on Ti6Al4V butt joints. In particular a Central Composite Design (CCD) with the central point repeated three times has been investigated. In the CCD there are two factors (power and speed of the fiber laser) and five levels for each factor. This paper deals with the investigation about the correlation between the severity of the undercut and the process parameters of the laser welding. In particular, through the confocal microscopy, the original geometry of the joint was accurately acquired and rebuilt in order to make a FEM model and simulate the mechanical behavior using Ansys14.5. Moreover, response surfaces and level curves were carried out to understand and predict the depth and the width of the undercut starting from the power and the speed of the laser. At last a mathematic and geometry regression was performed in order to find a unique conical curve that interpolates all the different undercuts and that varies its parameters according to the process parameters. It is established that the process with higher speed minimizes and optimizes the undercut in the joints.
\end{abstract}

\section{INTRODUCTION}

Titanium is one of the mostly used materials in the aeronautical field either because its excellent mechanical properties and its high strength to weight ratio. According to their microstructure, titanium alloys have been conventionally classified in three categories: $\alpha$ alloys, $\beta$ alloys and $\alpha+\beta$ alloys. Titanium alloys have low thermal conductivity (less than $22 \mathrm{~W} \mathrm{~cm}^{-1} \mathrm{~K}^{-1}$ ) that prevents the heat dissipation as well as a low coefficient of thermal expansion (about $8.6 x^{-6} \mathrm{k}^{-1}$ ) that prevents the appearance of stress during welding $[1,4,12]$. Titanium alloys also exhibit a good absorption rate of the laser beam (0.4\%) and a high melting temperature (about 1670 ${ }^{\circ} \mathrm{C}$ ), and then this means that a high energy must be used to weld these alloys. For these reasons, the key-hole laser welding is an effective technology to the processing of these alloys, since it allows a high location and a smaller melting zone, reducing the amount of energy required in comparison to other welding technologies [1, $3,6]$. The disadvantage of key-hole laser welding is the occurrence of the undercut defect. A profile of the weld bead acceptable should not present the undercut to have reliable fatigue properties [13]. The American Welding Society (AWS) defines the undercut as: "A condition in which the groove due to the welding, the welded surface in both the top and the bottom, is located below the surface of the base material adjacent" [2]. During the welding process different phenomena occur whose effects contribute to the formation of the undercut. In particular the physical principles to the base of these phenomena are:

- Evaporation and expulsion of the melted materials, due to the action of the high power density. The evaporation of the material is always present during a laser welding (LBW), while the ejection of material 
occurs when the vapor pressure in the keyhole (capillary steam) grows above a certain level, as discussed in [3] and [4]. As the laser beam moves forward, the undercut forms if the molten material cannot fill all the depression formed by the expulsion of the metal. This is the so-called spattering phenomenon. It is note that by increasing the specific heat input, expulsions increase and consequently the undercut enlarges.

- The contact angle to the edge of the weld depends on the surface tension at the interface between the phases. The temperature of the surface of the melting bath is significantly higher at the center than on the edge. The value of the contact angle is determined by the synergy of the surface tension in the molten material and of the laser beam and the spatial distribution of the power density [5]. Furthermore, the surface tension gradient induces the convection of Maragoni into the molten bath. The convection of Maragoni says that the liquid of the molten bath moves from the region at lower surface tension to the region at higher surface tension $[5,6]$. By modifying the surface tension, the angle of contact varies as well as the overall behavior of the molten alloy can be drastically changed.

- Transformation of phase implies the variation of the specific volume of the phases involved. The transition phase of the alloy Ti6Al4 from solid (density at room temperature $\approx 4.42 \mathrm{~g} / \mathrm{cm}^{3}$ ) to liquid (density at melting point $\approx 3.8 \mathrm{~g} / \mathrm{cm}^{3}$ ) causes an increase in specific volume of about $16 \%$. This means that the mass of the liquid cannot fall within the original volume but goes outside of the surface of the base material on both the top surface and the bottom. Of course, the solidification and cooling of the molten area implies a contraction thus having the opposite effect on the material of the weld pool [7].

The undercut results in a stress concentration at the edges of the weld and strongly influences the performance of the joints. The AWS suggests, in fact, that the maximum value of the undercut for welded joints used in aerospace must be at most $7 \%$ of the total thickness of the sheet metal to a welding class $\mathrm{A}$. The fatigue life is influenced by the value of the radius of the undercut, as reported in $[8,9,10]$. For this reasons it is fundamental to investigate on such a defect especially to develop applications that must satisfy rigid requirements for structural safety [14]. The undercut is a defect unviable of the laser welding and for this motivation it is of fundamental importance to minimize it. In particular, in this work a method is proposed to predict the severity of the undercut as a function of the process parameters. It is also presented a geometric study in order to obtain a unique curve simulating the profile of this defect, in order to obtain a prediction of the values characterizing the curve and consequently the undercut.

\section{MATERIALS AND METHOD}

In this study, butt-joint welding of square shaped $(40 \mathrm{~mm} \times 100 \mathrm{~mm} \times 3 \mathrm{~mm})$ Ti6Al4V samples (with mechanical properties given in Table 1) was carried out by using an ABB robot equipped with a Yb-fiber laser source (IPG Photonics YLR-3000) at SITEC, Laboratory for Laser Applications of the Department of Mechanical Engineering of Polytechnic of Milan. Equipment and constant parameters during the experimentation are listed in Table 2 . The specimen surfaces were chemically cleaned prior to welding (to eliminate surface contamination). To protect the weld pool and heated surfaces against the harmful influence of atmospheric air, top and bottom surfaces of joints were protected by high purity argon flow. To investigate the effect of the laser welding parameters on the weld geometry and specially undercut, the welds were produced at different welding speed and laser power according to the Central Composition Design listed in Table 3. In Fig. 1 an example of the top and the bottom sides of welded samples are shown.

\begin{tabular}{crl}
\hline Parameter & Value & \\
\hline Ultimate Tensile Strength (UTS) & 950 & MPa \\
Yield Strength $\left(\sigma_{y}\right)$ & $\mathbf{8 8 0}$ & MPa \\
Young's Modulus (E) & 113.8 & GPa \\
Poisson's Ratio (v) & $\mathbf{0 . 3 4 2}$ & \\
\hline
\end{tabular}

TABLE 1. Mechanical properties of Ti6A14V Annealed 


\begin{tabular}{|c|c|c|}
\hline & Parameters & Values \\
\hline \multirow{5}{*}{ Auxiliary gas } & Protection gas on the lower surface & Argon \\
\hline & Shielding gas flow on the lower surface $(\mathrm{L} / \mathrm{min})$ & 30 \\
\hline & & 30 \\
\hline & Protection gas on the upper surface & Argon \\
\hline & Gas flow rate of protection on the upper surface $(\mathrm{L} / \mathrm{min})$ & 55 \\
\hline \multirow{7}{*}{$\begin{array}{c}\text { Laser source and } \\
\text { system }\end{array}$} & & 3 KW Yb-Fiber \\
\hline & Laser Source & Laser \\
\hline & Fiber Diameter $(\mu \mathrm{m})$ & 400 \\
\hline & Laser Wavelength (nm) & 1070 \\
\hline & Beam quality factor $M^{2}$ & 6.606 \\
\hline & Focus position (mm) & $\mathbf{0}$ \\
\hline & Robot & $\begin{array}{c}\text { ABB IRB } 2400 \\
\text { robot }\end{array}$ \\
\hline \multirow{3}{*}{$\begin{array}{c}\text { Laser welding } \\
\text { head }\end{array}$} & Focal length of focus lens (mm) & 200 \\
\hline & Focal length of collimator lens (mm) & 100 \\
\hline & Spot Diameter $(\mu \mathrm{m})$ & 800 \\
\hline
\end{tabular}

TABLE 2. Material specifications and tools of key-hole laser welding process

\begin{tabular}{ccc}
\hline $\begin{array}{c}\text { Name of the } \\
\text { specimen }\end{array}$ & \multicolumn{2}{c}{ Process Parameters } \\
Speed & $(\mathbf{m m} / \mathbf{s})$ \\
\hline $1-2 / 1$ & 2750 & 30 \\
$2 / 1$ & 2700 & 35 \\
$3 / 1$ & 2850 & 35 \\
$4 / 1$ & 2950 & 40 \\
$5 / 1$ & 2850 & 45 \\
$6 / 1$ & 2850 & 35 \\
$7 / 1$ & 2850 & 25 \\
$8 / 1$ & 3000 & 35 \\
$9 / 1$ & 2850 & 35 \\
$12 / 1$ & 2950 & 30 \\
$13 / 1$ & 2750 & 40 \\
\hline
\end{tabular}

TABLE 3. Nomenclature and process parameters of the specimens (Experimental plan CCD)

\begin{tabular}{ll}
\hline $\begin{array}{c}\text { Sample } \\
\text { No. }\end{array}$ & Top and rear sides of surface \\
\hline & \\
\hline $1-2 / 1$ & \\
\hline
\end{tabular}

FIGURE 1. Top and rear sides of one of the welded samples

The following procedure for the study of the morphology of the samples was used:

1. Detection of the surface through Confocal Microscope Leica DCM3D

2. Leveling and filtering of the surface through the software LeicaMap to get characteristic measures of the joints

3. Elaboration of the .dat file and actual reconstruction through the software Solidworks 2013 and Ansys 14.5, in order to get the Kt of the joints

4. Parametric study of the surface of the joints in order to obtain a predictive geometry of the undercut across the surfaces of response and of the level curves

5. Convergence verification through experimental data present in literature

Through the software LeicaMap, the upper and lower surfaces of the joints were obtained. Then both upper and lower surfaces had undergone leveling and filtering. These two operations are used to ensure a good level position and parallel surfaces and also to eliminate outliers. On the upper surface (see an example in Fig. 2) measurements on 5 different profiles were obtained to ensure a low standard deviation. In this way it can be considered the average values.

In particular, the following three attributes were measured (Fig. 3): 
B Depth of the undercut (hollow to the side of the weld

bead)

$\mathrm{C}$

Distance between right and left undercut

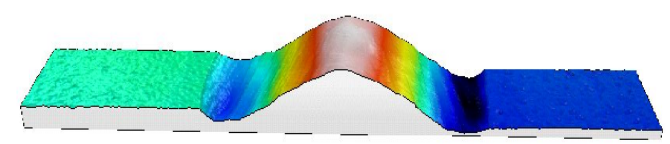

FIGURE 2. Elaboration of the upper surface of the welding through the LeicaMap software

After the reconstruction of the geometry of the joints through the programs Solidworks2013 and Ansys14.5, a tensile test was simulated to obtain the notch coefficient $\mathrm{Kt}$ in correspondence of the undercut. In particular, the mesh consists of Solid186 elements and different surfaces have been developed: the area of the weld bead, the side areas relative to undercuts, and the lateral areas of the base material. The different zones have been connected together via the contact elements of the bonded type in order to simulate the continuity of the material (as shown in Fig. 3.a). A tensile stress was applied with three different loads, as shown in Tab. 4. In particular, all the applied loads are lower than the yield strength and the variation of the loads serves to guarantee the independence between loads and results of the obtained Kt.
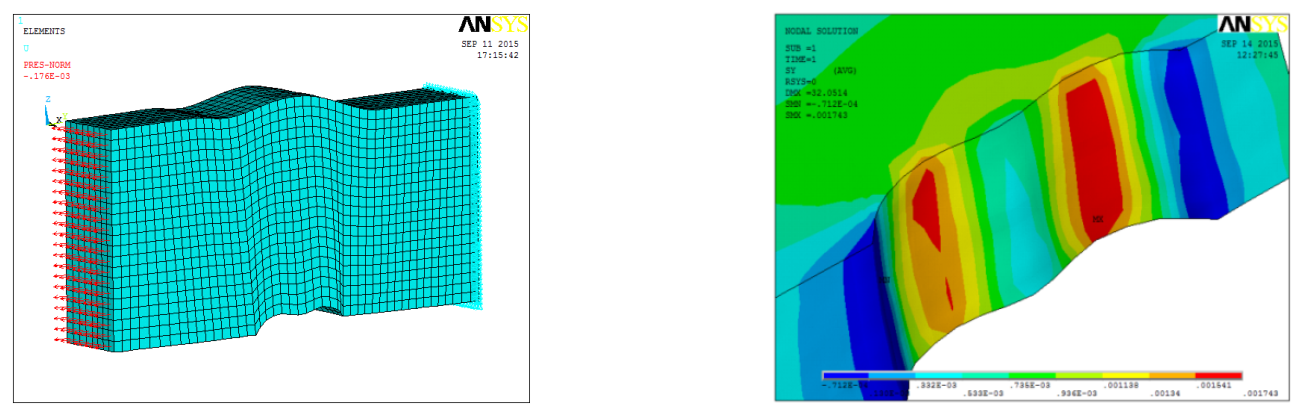

FIGURE 3. 3.a- Reconstruction of the numerical model of the joint, 3.b- Concentration of the stress in the lower surface in correspondence of the undercut

\begin{tabular}{ccc}
\hline & Load & {$[\mathbf{M P a}]$} \\
\hline $\mathbf{A}$ & $20 \% \sigma_{y}$ & 176 \\
$\mathbf{B}$ & $80 \% \sigma_{y}$ & 704 \\
$\mathbf{C}$ & $(\mathrm{b}-\mathrm{a}) / 2$ & 264 \\
\hline
\end{tabular}

TABLE 4. List of the loads applied to the numerical model

The points of the undercut profiles, along to the entire length of the specimen have then been reported in a single plane. The approximation of both right and left side of the weld bead has been calculated as depicted in Fig. 4. A quadratic regression analysis was performed to obtain the coefficients $a, b, c$ of the curve. The computational calculations were performed on an Intel ${ }^{\circledR}$ Core TM i7-3610QM CPU using Matlab R2012b 64bit.
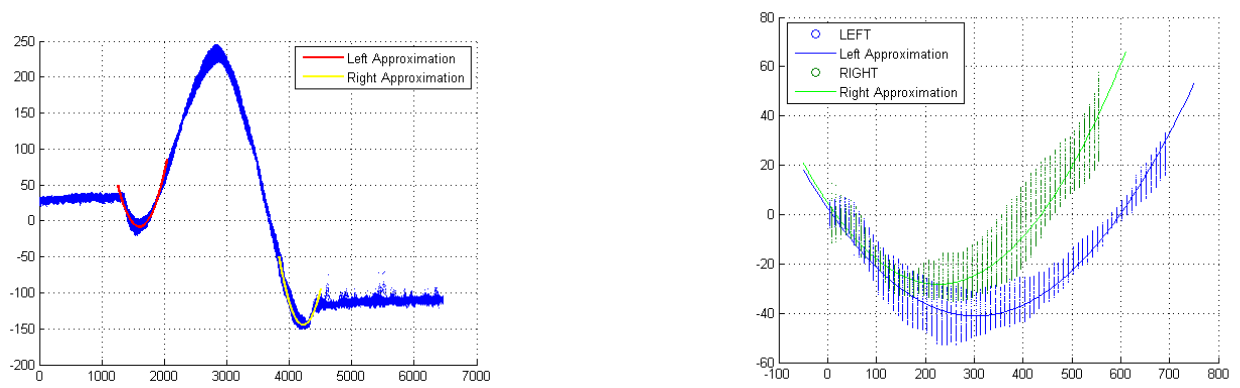

FIGURE 4. 4.a Evaluation of a unique curve that can fit all the undercut profiles of all the joints 4.b Average curve relative to all left and right profiles of the undercut 
In the present case starting from experimental plan $\mathrm{CCD}$, replicated twice, different response surfaces have been produced. In this case, the $\mathrm{k}$-factors are two, i.e the parameters of the welding process: speed $\mathrm{v}$ and power $\mathrm{P}$, which respectively represent the input values $\mathrm{x}$ and $\mathrm{y}$. Through the "Curve Fitting" tool of the software Matlab R2012b 64-bit the response surfaces and the level curves were plotted.

\section{EXPERIMENTAL RESULTS}

In tab. 5, for the sake of brevity, the better and the worst result concerning depth of undercut are reported. In particular the parameters, obtained from the measurement, are correlated with the process parameters (speed and power of the laser). The B value can be considered as a measure of the severity of the notch and it can be noticed that the specimen that get a better behavior (little value of B) is specimen 5. This sample had undergone the maximum speed value and a medium value of the power.
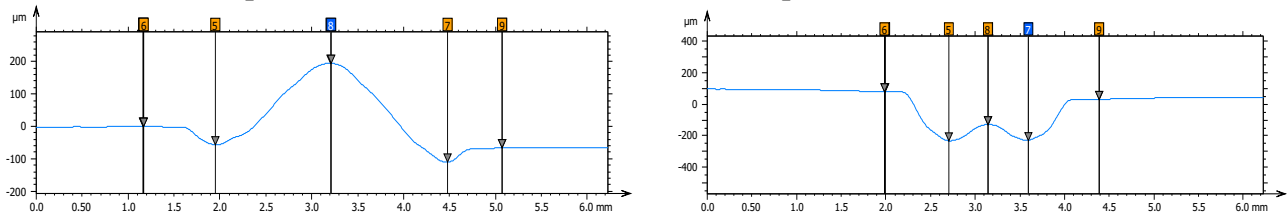

FIGURE 5. Measure on the upper and lower profile of the parameters: A (maximum height of the weld bead), B (depth of the undercut), C (distance between right and left undercut)

\begin{tabular}{cccccccccc}
\hline & \multicolumn{2}{c}{$\begin{array}{c}\text { Process } \\
\text { Parameters }\end{array}$} & \multicolumn{3}{c}{ Upper surface } & \multicolumn{3}{c}{ Lower surface } \\
\hline & P & S & A & B & C & A & B & C \\
5 & 2850 & 45 & 142.88 & 73.75 & 2.06 & 60.24 & 64.05 & 0.70 \\
7 & 2850 & 25 & 502.83 & 0.20 & 4.45 & 426.83 & 483.50 & 1.02
\end{tabular}

TABLE 5. Average measurements on the different profiles of the samples: $A$ is the maximum height of the weld bead, B stands for the depth of the undercut and $\mathrm{C}$ is the distance between the two undercuts

In Fig.3.b an image from the numerical model is reported where it is possible to understand that the most urged point is in correspondence of the undercut on the lower side. These results have been reported in tab. 6 and they have been compared with experimental data present in literature [10,15]. In fact, it has been noted that the point of maximum weakness, both in static and in dynamic tests, of a laser welded joint, is represented by the part adjacent to the weld bead in the lower zone of the joint. This point coincides with the undercut of the bottom surface. Also in this model it can be noted that the lower value of Kt belongs to sample 5. This fact confirms the coherence between the actual measurement on the surfaces and the numerical model. In Tab.6 the lower and the higher Kt are reported. These values confirm the agreement with the measurement obtained through the confocal microscopy.

\begin{tabular}{rccc}
\hline Sample & $\mathbf{P}$ & $\mathbf{S}$ & $\boldsymbol{K}_{\boldsymbol{t}}$ \\
\hline $\mathbf{5}$ & 2850 & 45 & 2,08 \\
$\mathbf{7}$ & 2850 & 25 & 3,09 \\
\hline
\end{tabular}

TABLE 6. Notch coefficient for the sample that experienced the lower and the higher speed of the laser

The acquired profile of the joint surface, the undercut in particular, offers the chance to try to describe the undercut itself via a curve with a known equation. In this case, it has been found that the curve that describe better the profile of the undercut is a parable:

$$
y=a x^{2}+b x+c
$$

The level $y=0$ for the parables is considered as the average of the height of the points immediately to the left (right) of the undercut. The level $\mathrm{x}=0$ for the parables was chosen for each case where the depression of the undercut starts. For the sake of brevity Fig. 6 it reports only a histogram giving the average value of the "a parameter", curvature as a function of the process parameters. The profiles of the undercut to the right of the weld bead were processed after apportioning points with respect to the vertical axis and then the base material goes towards the welding. In this configuration the comparison between the two sides and the calculation of an average parable for each specimen are easier. Tab.7 reports all the values relative to all the parables factors. 


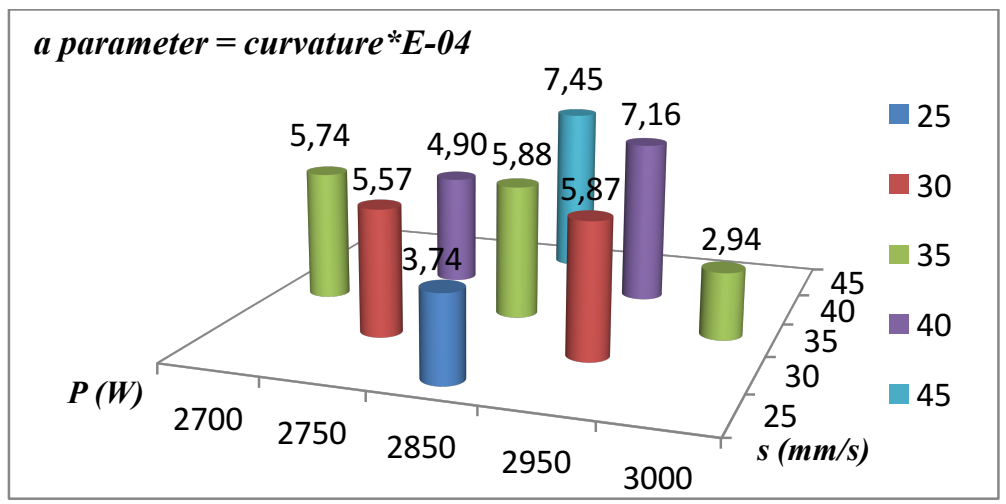

FIGURE 6. Histogram representation of the variation of the average a parameter of the parable (curvature)

The most significant surfaces and curves for the purpose of this work are reported and they are in function of the depth of the notch on the lower surface (Bdown) and the coefficient of notch Kt (see Fig.7 and Fig.8). The parameter used to measure the goodness of the approximation is the coefficient of determination Rsquare. The type of fitting used is a second degree polynomial for both $\mathrm{x}$ and $\mathrm{y}$, and then the mathematical model obtained is of type:

$$
f(x, y)=\beta_{00}+\beta_{10} x+\beta_{01} y+\beta_{20} x^{2}+\beta_{11} x y+\beta_{02} y^{2}
$$

The surfaces of response represent the $\mathrm{z}=\mathrm{f}(\mathrm{x}, \mathrm{y})$ function where $\mathrm{z}$ is chosen time to time between the parameters calculated from the preceding analyzes (Kt and Bdown). With the response surfaces but above all with the level curves, it is clear that $\mathrm{Kt}$ coefficient reduces with the increasing of the speed without a significant influence of the power of the source and that Bdown reduces by coupling high speed with medium and higher power.

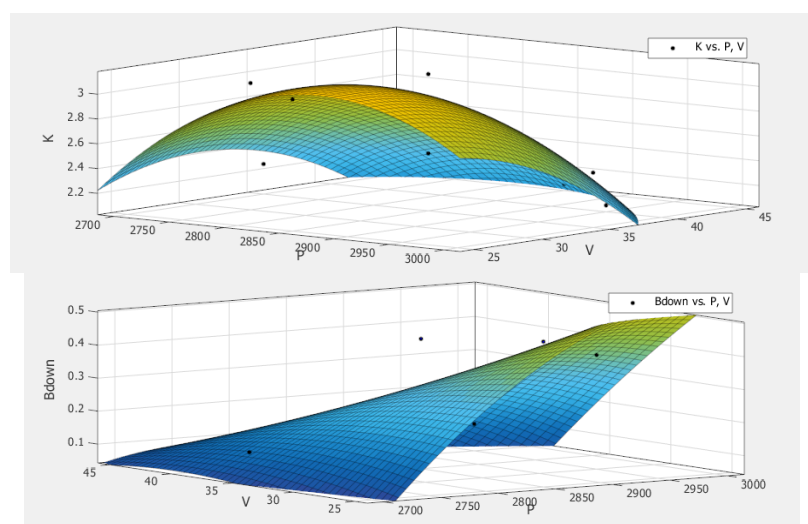

FIGURE 7. 7.a Response surface relatives to Kt $(\mathrm{R}-\mathrm{Square}=0.78)$, 7.b Response surface relatives to Bdown parameter $(\mathrm{R}-\mathrm{Square}=0.8)$
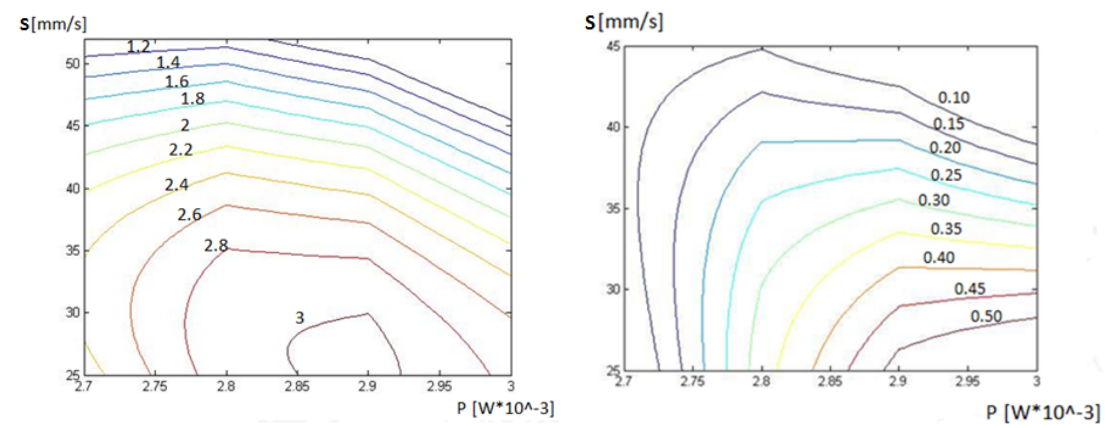

FIGURE 8. 8. a Level curve relatives to Kt, 8.b level curve relatives to the parameter Bdown 


\section{CONCLUSION}

The aim of this study was to find a predictive method capable to control and minimize the effect of the undercut as a function of the process parameters of the laser. It is also presented a geometric study to obtain a unique curve that can simulate the profile of this defect. Thanks to the level curves and to the prediction of the profile geometry the severity of the undercut has been evaluated and linked to the laser parameters, i.e speed and power. On the basis of the experimental results presented and discussed in the previous sections the following considerations can be drawn:

- The faster the welding speed the smaller the depth of the dip and the smaller value of the notch factor Kt

- The parable has been found to be the curve that describe better the undercut profile. For each couple of speed and power of the laser, a given set of parable coefficients has been found.

- The curvature of the parable reduces with the increasing of the speed and medium and high power of the laser.

In conclusion thanks to a control between the numerical result obtained and the experimental one present in literature, it is possible consider the level curves and the response surfaces as a plan in order to minimize the undercut, which is inevitable on the welding laser.

\section{References}

[1] A. Astarita, C. Testani, F. Scherillo, A. Squillace, L. Carrino, Beta Forging of a Ti6A14V Component for Aeronautic Applications: Microstructure Evolution, Metallogr. Microstruct. Anal., 3 (2014) 460-467

[2] AWS A3.0:2001., Standard welding terms and definition, including terms for adhesive bonding, brazing, soldering, thermal cutting, and thermal spraying, An American National Standard, Approved by American national Standards Institute, American Welding Society, Miami, FL (2001) 41

[3] M. Pastor, H. Zhao, R.P. Martukanitz, T. Debroy, Porosity, underfill and magnesium loss during continuous wave Nd:YAG laser welding of thin plates pf aluminum alloys 5182 and 5754, Weld J., (1999) 78207-s-216-s [4] C. Dawes, Laser Welding, McGraw-Hill, Inc., New York, Usa, (1992) 73

[5] B.J. Aalderink, D.F. de Lange, R.G.K.M. Aarts, J. Meijer, Keyhole shapes during laser welding of thin metal sheets, J. Phys. D:Appl. Phys. ,40 (2007) 5388-5393

[6] R. Fabbro, Melt pot and keyhole behavior analysis for deep penetration laser welding, J. Phys. D:Appl. Phys. 43 (2010) 445-501

[7] A. Squillace, U. Prisco, S. Ciliberto, A. Astarita, Effect of welding parameters on morphology and mechanical properties of Ti-6Al-4V laser beam welded butt joints, Journal of Materials Processing Technology, 212 (2012) 427-436

[8] T.N. Nguyen, M.A. Wahab, The effect of welding geometry and residual stresses on fatigue of welded joints under combined loading, J. Mater. Process. Technol., 77 (1998) 201-208

[9] W. Fricke, Fatigue analysis of welded joints: state of development, Mar. Struct., 16 (2003) 185-200

[10] X. Cao, M.Jahazi, Effect of welding speed on butt joint quality of Ti-6Al-4V alloy welded using a highpower Nd:YAG laser, Optics and Lasers in Engineering, 47 (2009) 1231-1241

[11] J.D. De Stefani, Introduction to titanium and titanium alloys ASM Handbook, vol 2, Materials Park, Ohio, USA, ASM International (1990) pp. 586-591

[12] K. Richter, W. Behr, U, Reisgen, Low heat welding of titanium materials with a pulsed Nd:YAG laser, Material Wiss Werkst 38(1) (2007) 51-56

[13] Zhang Li, S.L. Gobbi, I. Norris, S. Zolotovsky, K.H. Richter, Laser welding techniques for titanium alloy sheet, J. Mater. Process. Technol. (1997) 203-208

[14] C. Casavola, C. Pappalettere, F. Tattoli, Experimental and numerical study of static and fatigue properties of titanium alloy welded joints, Mechanics of Materials, 41 (2009) 231-243

[15] X.L. Gao, L. J. Zhang, J. Liu, J.X. Zhang, A comparative study pulsed Nd:YAG laser welding and TIG wleding of thin Ti6Al4V titanium alloy plate, Mater. Sci. Eng. A., 559 (2013) 14-21. 